\title{
Praktische Philosophie und Unternehmensethik \\ Weisheit und Wirtschaft: Konstruktiver Dialog oder Kategorienfehler?
}

\author{
INA SCHMIDT ${ }^{*}$
}

\section{Philosophy and Ethical Business - A Dialogue between Wisdom and Economy: Profitable Alliance or Twisted Categories?}

The idea of a philosophical view to open new economic perspectives on values and social responsibility creates new possibilities within the debate about etbical standards in the context of companies and organizations. Through defining and describing the structures of thinking and communicating in economic systems and especially in complex situations of change, a philosophical dialogue helps to clear the real situation and the real possibilities of acting - in an ethical and responsible way: authentic and open minded.

Keywords: Individualetbik, Unternehmenswerte, Handlungsspiel und -freiraum, Selbstverantwortung, Beratung als offenes Konzept

Martin Heidegger (1951-52: 8) hat der Wissenschaft vor über fünf Jahrzehnten einmal vorgeworfen, sie denke nicht, Ähnliches hätte er vermutlich über die Wirtschaft der Gegenwart gesagt. Die Vertreter der damaligen Wissenschaft waren entsetzt, die heutigen Lenker großer Konzerne wären es mit Sicherheit auch. Was aber ist gemeint?

Auch wenn wir gegenwärtig eine sehr lebendige und teilweise hitzige Debatte führen, wie sich ethische Werte in ein unternehmerisches Handeln integrieren lassen, scheint es sich doch immer noch auszuschließen, dass wirtschaftliches Handeln etwas mit praktischer Philosophie zu tun haben könnte. Ist die Ethik, die die Wirtschaftsethik meint, etwas ganz und gar Unphilosophisches und wenn ja, warum sollte das so sein?

Das Denken, das die praktische Philosophie anstrebt, ist ein Denken, das sich der Realität annimmt, wie sie sie vorfindet. Sie übt sich in einer ,voraussetzungslosen Wahrnehmung“" (vgl. dazu den Kern des phänomenologischen Denkansatzes bei Husserl 1901) und lässt so möglicherweise zunächst ein verwickeltes Gebilde von Fragen und Problemstellungen sichtbar werden - nicht entstehen! -, die einer schnellen Beantwortung des Problems bzw. häufig nur des Symptoms im Wege stehen. Damit ist die Philosophie aber alles andere als ineffizient, denn im Gegensatz zum verbreiteten Set von Standardtools, das im Beraterkontext gerne verwendet wird, versucht sie ihr

\footnotetext{
Dr. Ina Schmidt, Kulturwissenschaftlerin und praktische Philosophin, Inhaberin der denkraeume, einer philosophischen Beratung in Reinbek bei Hamburg, Kreutzkamp 57a, D-21465 Reinbek, Tel.: +49-(0)40-72813746, E-Mail: ina.schmidt@denkraeume.net, Web: www.denkraeume.net, Forschungsschwerpunkte: Lebensphilosophie seit der Frühromantik in ihren Auswirkungen auf die Philosophie des 20. Jahrhunderts - exemplarisch am Frühwerk Martin Heideggers, Kulturphilosophie und Hermeneutik mit Schwerpunkt Wilhelm Dilthey und Georg Simmel, gegenwärtig Arbeiten zur Übersetzung philosophischer Theorien auf aktuelle Beratungskontexte.
} 
Denken den Gegebenheiten anzupassen und nicht die Gegebenheiten ihrem geistigen Werkzeugkoffer. Ersteres ist aber ohne Alternative, wenn hochgesteckte Ziele wie Nachhaltigkeit und gesellschaftliche Verantwortung ernsthaft und wahrhaftig verfolgt und nicht als PR-Instrument verurteilt werden wollen. Denn alles, was den Blick auf die reale Situation mit ihren tatsächlichen Mängeln und Grenzen, vermeidet, bleibt Fassade und verwechselt das ethische Anliegen mit einer ergebnisorientierten Imagepflege.

Diese Unterscheidung ist mittlerweile auch in Unternehmenskreisen angekommen. Allerdings werden daraus häufig seltsame Schlussfolgerungen gezogen: Nicht die eigene Struktur wird zum Gegenstand eigenen Nachdenkens, sondern der Blick richtet sich nach draußen: Wer macht das, was wir nicht wollen, weil wir glauben, dass wir es nicht dürfen oder können? Welches Hilfsprojekt lässt sich fördern? Welchen Kindergarten können wir unterstützen und wo können wir als Sponsor einer ehrenwerten Veranstaltung auftreten - wie also können wir von einem „Dritten“ profitieren? Es entsteht zwar eine Art Kooperation, die aber weder eine echte Partnerschaft bedeutet, noch wirkliche Veränderung anstrebt, sondern das soziale Marketing im Vordergrund stehen lässt: eine verkappte „Win-Win“-Gemeinschaft, nicht weniger, aber auch nicht mehr.

Der Gedanke einer philosophischen Beratung lehnt ein solches Vorgehen ab. Der Weg zu effizienten Strukturen, zu stabilen Profiten kann nur über die optimale Ausrichtung und Auslastung dessen gegangen werden, was das Unternehmen selbst an Ressourcen und Fähigkeiten in sich versammelt: Es wäre immer schön, wenn es anders wäre, vielleicht sogar besser, aber es gilt eben mit dem umzugehen, was da ist. Was sehr banal klingt, ist möglicherweise die größte Herausforderung einer ethischen Ausrichtung - persönlich wie unternehmerisch. Unternehmensethik und CSR ist damit nicht Aufgabe einer einzelnen Stabsstelle oder Abteilung, sondern eine individuelle Herausforderung, die Teil einer tatsächlich gelebten Ethik-Charta oder neudeutsch eines „Code of Conduct“ sein muss. Sollten diese Maßgaben nur Kopfgeburten einer bilanzorientierten Vorstandsetage sein, werden sie nicht nur überflüssig, sondern im Gegenteil sogar destruktiv.

Um dem entgegenzuwirken ist eines unerlässlich: der persönliche Dialog, der den individuellen Freiraum schafft, und damit eine Plattform für Fragen, Zweifel, eigene Gedanken und möglicherweise versponnene Antworten bietet (vgl. dazu das Verständnis eines „tätigen Denkens“ als höchste Form des Handelns bei Hannah Arendt 1958: $361 \mathrm{ff}$.). Dies ist immer der erste Schritt, ob im Einzeldialog oder einem internen Workshop. Nur aus dieser wahrhaftigen Auseinandersetzung entsteht das Material, das notwendig wird, um den Kernthemen auf die Spur zu kommen. In einem solchen Gespräch haben sich schon verschiedene Führungskräfte darüber gefreut, dass ihre Gedanken nicht immer ein Ergebnis haben mussten, um dann festzustellen, dass genau diese Erwartung, die sie an das eigene Denken stellen, das Kernproblem ihres gesamten Teams abbildet. Kreativität und Innovation entstehen nicht, wenn die Antwort schon vor der Frage feststeht. Meist folgt dann aber gleich die nächste Sorge: Wenn ich diesen „Spielraum“ zulasse, um Begriffe zu klären und mein eigenes Denkgebäude zu durchleuchten, was passiert dann mit meinen Überzeugungen, mit dem 
vermeintlich Richtigen und Falschen? Wie stehe ich als Führungspersönlichkeit da, wenn ich nicht immer genau weiß, wo es lang geht?

Diese Unsicherheit als selbstverständlichen Teil eines jeden Prozesses deutlich zu machen, ist ein wichtiger Ausgangspunkt der philosophischen Arbeit. Der Philosophie geht es darum, das „Richtige“ von seinem absoluten Anspruch zu befreien und das „Beste“ für den individuellen Kontext herauszuarbeiten: Die Frage nach den Instrumenten eines authentischen Führungsstils ist in einem Großkonzern eine andere als in einem inhabergeführten Meisterbetrieb. In einer Veranstaltung zur Frage nach der Verbindung von „Kommunikation und Führung“" standen sich ein Konzernmanager und eine Mitarbeiterin aus einem mittelständischen Unternehmen zu dieser Frage gegenüber. Sie konnten sich nicht einigen, sind aber beide auf neue Gedanken gekommen - und hatten beide recht.

In solchen Fällen kommt es darauf an, die Ausrichtung des Einzelnen zu klären seine ganz bewusst eingenommene Haltung, für die er sich entscheiden muss. In einem Coachinggespräch mit einer Führungskraft aus der IT-Branche entstand eine intensive Diskussion um die Möglichkeit einer solchen „objektiven“ Entscheidung. Kann eine eigene Haltung jemals objektiven Maßstäben genügen, kann es also - darüber hinaus - so etwas wie eine universale Ethik geben? Am Ende des Gesprächs hatte die vermeintlich notwendige Begründung einer getroffenen Entscheidung auf der Basis von objektiven Fakten eine völlig neue Bedeutung: Damit war nichts zerstört, sondern etwas anderes „,befreit" worden. Ähnlich reagierte der Geschäftsführer einer Firma mit etwa 50 Mitarbeitern nach einem Workshop zum Thema „Verantwortung und Komplexität": Seine eigene Kraft hatte sich darin erschöpft, Verantwortung für andere zu übernehmen und sich ein sehr komplexes Gebilde von Rollen und $\mathrm{Zu}$ ständigkeiten aufzubauen. Dass er auch eine Verantwortung sich selbst gegenüber trägt, war eine Erkenntnis, die nicht neu, aber vollständig verschüttet gewesen war. Dieses - mit Platon gesprochen - erinnerte Wissen führte zu völlig neuen Möglichkeiten und Ideen, die in das bisherige Geflecht nicht hineingepasst hatten.

Das Ergebnis philosophischer Beratungsgespräche ist damit eine andere Form der Klarheit, die dazu befähigt, bestehende Problemstellungen mit einer neuen Sichtweise anzugehen: individuell wie kollektiv. Was wäre eigentlich, wenn ...? Was verstehe ich eigentlich unter ...? Sich bzw. seine Abteilung oder sein Unternehmen selbst zum Thema zu machen, ist oft nicht einfach, aber im Umgang mit übergeordneten Themen und Zielen notwendig. Ein solches Denken und Handeln fängt zunächst beim Einzelnen an, kann aber durch Unternehmensstrukturen gefördert und sogar initiiert werden. Die einzelnen Mitarbeiter in einem Unternehmen wie der Drogeriekette „dm“ erfahren durch die persönlichen Überzeugungen ihres Gründers Götz Werner eine andere Unternehmenskultur (vgl. Werner: 115-145), eine andere Weltanschauung als die Manager eines Konzerns, dessen Ziel nur darin besteht, den eigenen Gewinn oder den des Vorstands - zu maximieren.

Unternehmen sind nichts anderes als ein organisches Kollektiv aus einzelnen Menschen, und es geht in der philosophischen Praxis darum, durch Veranstaltungen und Beratung Anstöße zu geben, die auf die Bedeutung der eigenen, persönlichen Haltung aufmerksam machen - zum Nutzen des Kollektivs. 
Wenn es also in diesem Sinne um eine Verknüpfung von Wirtschaft und Drittem Sektor geht, dann im Sinne eines gegenseitigen Lernprozesses, der sich auf die Stärken der jeweils anderen Seite bezieht: Wie lässt sich das, was die vielleicht idealistische Mission des einen ausmacht, in einen unternehmerischen Kontext übersetzen? Wie lässt sich umgekehrt von den klaren Strukturen eines Unternehmens etwas für die eigene „Idee“ übernehmen, damit sie auch tatsächlich in die Welt kommt? Bei der Begleitung des Leitbildprozesses einer Bildungseinrichtung wurde beispielsweise beim Blick in die Vergangenheit deutlich, dass die Idee und die Inhalte derart hoch gehängt waren, dass sie nicht mit etwas so Schnödem wie „Profit“ in Verbindung gebracht werden sollten. Hier herrschte also die gegenläufige Tendenz der reinen Gewinnorientierung, und es musste geklärt werden, was den „Erfolg“ für eine solche Institution denn eigentlich kennzeichnen solle. Was bedeutet Geld? Eine überaus philosophische Fragestellung.

$\mathrm{Ob}$ in persönlichen Einzelgesprächen, Workshops oder Diskussionsrunden zu solchen Themen, meldet sich an dieser Schnittstelle häufig der Irrglaube, man müsse sich für das eine oder das andere entscheiden: entweder die gute Idee oder das Geldverdienen! Warum lässt sich das eine nicht mit dem anderen verbinden, warum handeln wir erst „ehrenwert", wenn es der Leidensdruck nicht mehr anders möglich macht? Und ist es dann eigentlich noch ehrenwert? Welche Angst wovor ist an dieser Stelle der eigentliche Motor - auch wenn immer wieder Fakten und scheinbare Notwendigkeiten vorgeschoben werden?

Diese Fragen müssen nicht nur gestellt, sondern auch beantwortet werden, und das geht nur in einem - sokratischen - Dialog auf Augenhöhe, der einen eigenen (Zeit-) Raum bekommt und den einzelnen Mitarbeiter dabei ebenso ernst nimmt, wie die Ausrichtung des Unternehmenskollektivs. Dabei stehen diesem Ansatz zwei Dinge gerne und häufig im Weg:

(1) Erstens, der Zeitfaktor: Ein solches Vorgehen braucht Zeit, aber dieses Argument ist vielfach durch eine Aufstellung der Zeiten auszuhebeln, die durch die falschen Fragen und damit ineffizienten Maßnahmen entstanden sind. Dennoch braucht es hier ein neues Bewusstsein für den Wert langfristiger Maßnahmen.

(2) Zweitens, und dieser Punkt sitzt tiefer und betrifft unser grundsätzliches Denkgerüst: Wir denken - gerade in wirtschaftlichen Zusammenhängen - fast ausschließlich in Dualismen und behindern damit oft schon im Ansatz eine wirklich fruchtbare Zusammenarbeit der Disziplinen: der Wirtschaft mit der Ethik, der Naturwissenschaft mit der Geisteswissenschaft, des Controlling mit der CSR-Abteilung. Hier sollten wir unser Augenmerk verstärkt darauf richten, wie wir mit Unterschiedlichkeit umgehen können, um die daraus entstehende Vielfalt als bereichernde Quelle anzuzapfen.

Bernhard von Mutius (2004: 14f.) hat in seinem sehr schönen Buch „Die andere Intelligenz" einführend geschrieben, wir sollten wieder lernen, das Verbindende (gerade innerhalb der Unterschiedlichkeit) zu sehen, bevor wir uns um das Trennende kümmern - diese Einsicht von der intellektuellen Erkenntnis in eine gelebte Erfahrung zu überführen, darum geht es der praktischen Philosophie im Sinne einer tatsächlichen Unternehmensphilosophie. Eine ernst gemeinte philosophische Praxis hat daher nichts mit kryptischen Bandwurmsätzen und verklärter Lebensferne zu tun, sondern 
lebt selbst von der Verknüpfung theoretischer Arbeit mit pragmatischen Anwendungsfällen.

Diese Verbindung findet auf unterschiedliche Weise statt, meist in Form von Seminaren, Workshops gemeinsam mit Kooperationspartnern aus dem Bereich Nachhaltigkeitsmanagement und Coaching oder im Rahmen von Vorträgen oder Podiumsdiskussionen zu Kernthemen wie bspw. der Klärung von Entscheidungsprozessen oder dem Verhältnis von Komplexität und Verantwortung. Fragen zu dem, was eine ,gute“ Führung ausmacht oder was soziale Gerechtigkeit bedeutet, lassen sich aus philosophischer Sicht um Perspektiven bereichern, die dem reinen Wirtschaftskontext nicht zur Verfügung stehen. Hier entwickelt sich häufig eine sehr lebhafte Debatte um die Möglichkeit eines individualethischen Ansatzes (vgl. dazu den Begriff des individuellen Gesetzes bei G. Simmel 1968: 174ff.) für eine wirtschaftsethische Ausrichtung, und oft entsteht aus vehementem Widerstand dann ein sehr persönliches Nachdenken.

Und genau darin liegt die Antwort auf die von Martin Heidegger aufgestellte These: Das Anwenden mechanistischer Theorien und Regelsetzungen ist notwendig - für die persönliche, die wissenschaftliche wie auch die unternehmerische Ausrichtung -, sogar lobenswert, aber es ist eben kein selbständiges Denken. Denn dieses Denken bedeutet keine luxuriöse Selbstbespiegelung, sondern bildet das eigentliche Fundament, auf dem sich pragmatische Strukturen und Prinzipien erst etablieren können. Aus einer solchen Verbindung entsteht dann häufig nichts weniger als persönlicher und unternehmerischer Erfolg.

\section{Literaturverzeichnis}

Arendt, H. (1958/1998): Vita activa. Oder Vom tätigen Leben, München.

Heidegger, M. (1951-1952/1992): Was heißt Denken?, Stuttgart.

Husserl, E. (1901/1992): Logische Untersuchungen, Bd. 1: Prolegomena zur reinen Logik, Hamburg.

Merleau-Ponty, M. (2003): Primat der Wahrnehmung, Frankfurt a. M.

Mutius, B. v. (2004): Die andere Intelligenz, Stuttgart.

Simmel, G. (1968/1987): Das individuelle Gesetz, Frankfurt a. M.

Werner, G. W. (2007): Einkommen für alle, Köln. 